\title{
Photo-, osmo- and phonophobia in the premonitory phase of migraine: mistaking symptoms for triggers?
}

\author{
Laura H Schulte, Tim P Jürgens and Arne May* \\ Please see related articles: http://www.thejournalofheadacheandpain.com/content/16/1/2 and http://www.thejournalofheadacheandpain.com/ \\ content/16/1/3
}

\begin{abstract}
Background: Certain environmental stimuli are frequently reported as typical triggers of migraine pain. Whether these so-called triggers are independent precipitators of migraine pain or mere symptoms of the premonitory phase of migraine remains to be elucidated.

Methods: In this retrospective cohort study of 1010 migraine patients of a tertiary headache center we assessed the frequency of common trigger factors, premonitory symptoms and accompanying symptoms as well as basic headache characteristics and demographic data.

Results: Premonitory symptoms with an onset of 2 or more hours prior to the headache were present in $38.9 \%$ of migraine patients, the most frequent being a tense neck, phonophobia and difficulty concentrating. There was a clear overlap of certain trigger factors and the presence of corresponding premonitory symptoms: flickering or bright light as a trigger was associated with higher frequency of photophobia in the premonitory phase. The same applied to the presence of food craving and osmophobia in the premonitory phase and certain foods or odours as trigger factors.

Conclusions: Our data thus support the view that commonly reported trigger factors of migraine are not so much independent precipitators of migraine pain, but that they are most likely just misinterpreted results of enhanced attention to certain stimuli mediated by typical premonitory symptoms of migraine pain.
\end{abstract}

Keywords: Migraine; Tipping point; Premonitory symptoms; Triggers; Photophobia; Phonophobia; Nausea

\section{Background}

Flickering light, bright sunlight, certain odours or certain foods are - among others - frequently reported as typical triggers of migraine pain [1-11]. However, empirical evidence that these factors can actually provoke migraine attacks is scarce [12,13], and it is currently not clear whether these factors are actual independent triggers of migraine pain, i.e. factors that at any given moment reliably precipitate migraine pain, or whether the increased intake of certain foods, the pronounced perception of e.g. certain lights or odours as adverse and even pain inducing might rather be a result of a slight photo- or osmophobia or food craving already present in the premonitory phase

\footnotetext{
* Correspondence: a.may@uke.de

Department of Systems Neuroscience, University Medical Center Hamburg-Eppendorf, Martinistr. 52, Hamburg D-22046, Germany
}

of migraine. It is therefore possible that these factors are rather a symptom than a true trigger of migraine [14,15]. To further elucidate this problem we analyzed the data of 1010 migraine patients.

\section{Methods}

In this retrospective cohort study, 1805 patients of a tertiary headache center (Headache Outpatient Department of the University Medical Center Hamburg-Eppendorf) upon first admission were presented with a variety of tests and custom questionnaires presented in electronic form between November 2009 and July 2014. During this first admission patients were seen and diagnosed by a trained headache specialist (TPJ, AM), following the IHS criteria. Patients with a diagnosis of migraine with or without aura were included in the study. 
As part of the battery of questionnaires, patients were asked for the presence or absence of 27 typical migraine premonitory symptoms (see Figure 1). In one additional question the latency between onset of the reported premonitory symptoms and the headache phase was assessed. As we suspected a tendency among patients to not clearly differentiate between aura symptoms or predominant accompanying symptoms of the early pain phase of migraine and premonitory symptoms, we only regarded symptoms as truly premonitory, if patients reported an onset at least two hours prior to the headache. Only these patients were included when investigating the association of premonitory symptoms and trigger factors. Additionally we assessed basic headache characteristics, including 21 accompanying symptoms of the headache as well as 24 different trigger factors, among these flickering light, bright sunlight, certain foods and odours (see Table 1).

Data collection in a local data base and use of the data for scientific analysis and publication was approved by the local ethics committee of the chamber of physicians of Hamburg, Germany (submission number PV3185). Informed consent was obtained from all participants. Data were analyzed using SPSS Statistics. Categorical

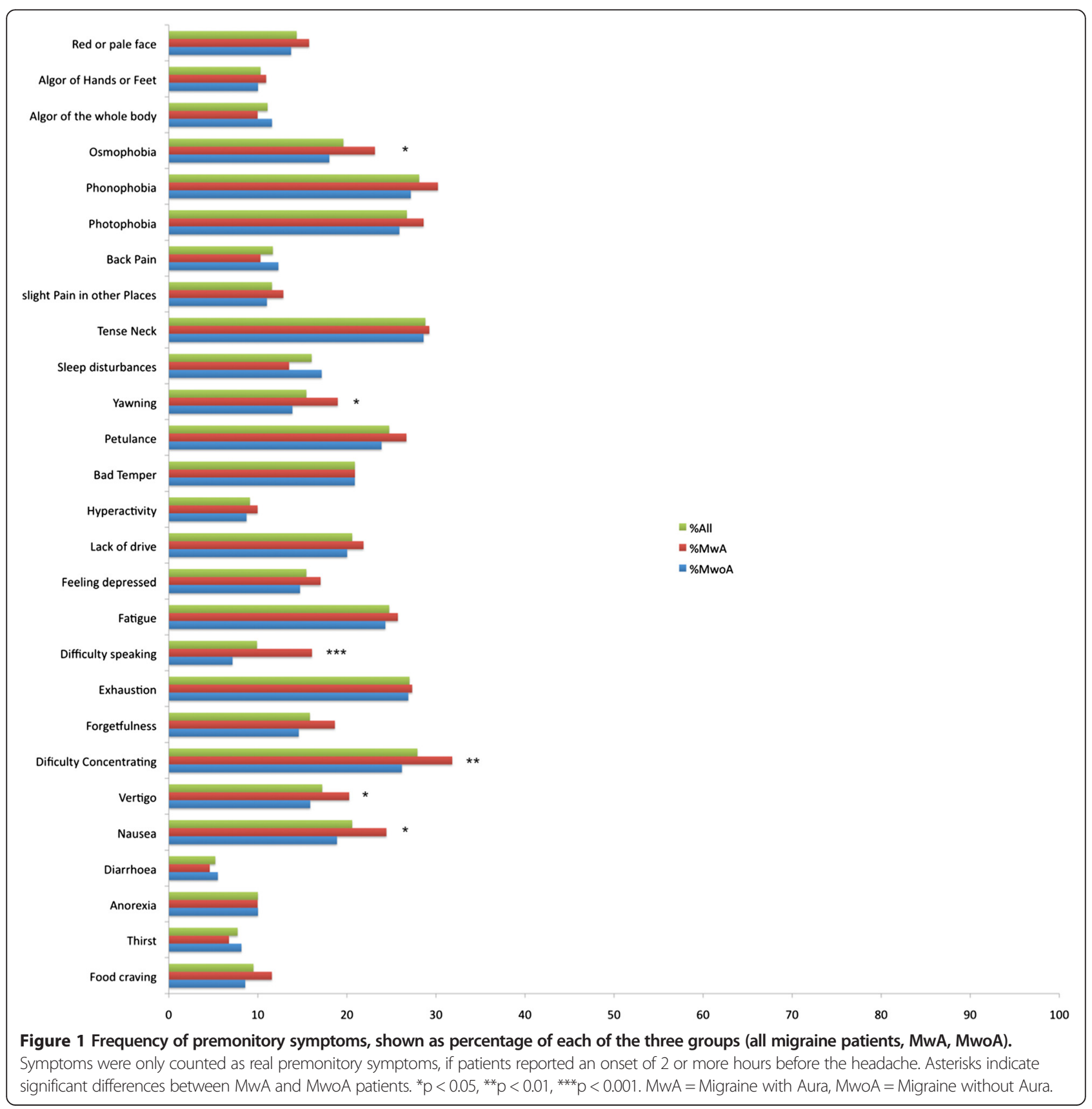


Table 1 Headache triggers and accompanying symptoms of headache assessed by our questionnaires

Headache triggers

Accompanying symptoms of headache
Foods, stress, relieve from stress, weather/change in weather, bright sunlight, flickering light, heat, cold, physical exercise, sexual intercourse, irregular meals/skipping of meals, decreased water intake, sleep abundance, sleep deprivation, holiday, alcohol, smells/odours, resolvents, electric fields, medication, hormonal changes, specific head movements, other illnesses (e.g. common cold), touch

Photophobia, phonophobia, osmophobia, need for rest, nausea, vomiting, allodynia of the skin, vertigo, facial rush, facial sweating, restlessness/agitation, swelling of lymph nodes, neck pain, conjunctival injection, tearing, eyelid oedema, ptosis, nasal congestion, rhinorrhoea, difficulty speaking, blurred vision variables were compared using chi-square-test or - in case of cell numbers smaller than 6 - Fisher's exact test. Continuous variables were analyzed using student's $t$ test or one way ANOVAs (in case of more than two groups). As the amount of missing data sets was low $(<1.5 \%)$, we did not apply a special correction technique.

\section{Results}

Of the 1805 headache patients presented with the questionnaires, 1010 patients were diagnosed with migraine. Of these, 699 had migraine without aura and 311 had migraine with aura (see Table 2 for demographic details of the study population). 389 migraine patients (resp. $38.5 \%$ of all migraine patients) reported premonitory symptoms starting at least 2 hours before the pain phase of migraine with no significant difference between MwoA and MwA (269 MwoA (38.5\%) and 120 MwA (38.6\%)). The 389 patients experiencing these phenomena reported an average of approximately 12 different premonitory symptoms with a mean onset of about 6.3 hours before the headache, the most frequent being a tense neck, phonophobia and difficulty concentrating (see Figure 1 for more detail). There were significant differences regarding the frequency of certain premonitory symptoms: Speaking difficulties, yawning, vertigo, nausea, osmophobia and difficulties concentrating were found significantly more often in MwA patients. There were significant but overall weak correlations between the sum of premonitory symptoms and age, sex, and MIDAS-Score.

When analyzing the interrelationship of reported trigger factors and corresponding premonitory symptoms, we found that migraine patients suffering from photophobia

Table 2 Demographic characteristics of the study population

\begin{tabular}{llll}
\hline & MwoA & MwA & Total \\
\hline Number of patients & 699 & 311 & 1010 \\
Age & $39.2 \mathrm{y}$ & $40.2 \mathrm{y}$ & $39.5 \mathrm{y}$ \\
Sex & $130 \mathrm{~m}, 569 \mathrm{f}$ & $51 \mathrm{~m}, 260 \mathrm{f}$ & $181 \mathrm{~m}, 829 \mathrm{f}$ \\
Headache days per month & 11.3 days & 9.9 days & 10.9 days \\
Disease duration & $12 \mathrm{y}$ & $14.4 \mathrm{y}$ & $12.7 \mathrm{y}$ \\
MIDAS-Score (Average) & 44.9 & 45.9 & 45.2 \\
\hline
\end{tabular}

in the premonitory phase of migraine pain reported flickering light or bright sunlight as a trigger factor significantly more often than patients not experiencing photophobia in the premonitory phase. Accordingly, odours as a migraine trigger were significantly more frequent among patients reporting osmophobia as a premonitory symptom. The same distribution applied to certain foods as a trigger and the presence of food craving in the premonitory phase ( $\mathrm{p}<0.0001$ for all comparisons). Additionally, in patients reporting nausea, photo-, osmo- or phonophobia in the premonitory phase these symptoms were significantly more frequent as accompanying symptoms of migraine pain ( $\mathrm{p}<0.0001$ for all comparisons).

\section{Discussion}

In the current study the prevalence rate of premonitory symptoms among migraine patients was $38.5 \%$. We found a huge overlap between certain so-called triggers and the respective corresponding premonitory and accompanying symptoms of migraine pain.

There are currently five larger scale studies investigating the frequency of premonitory symptoms in migraine which found percentages between $32.9 \%$ and $86.9 \%$ of migraine patients reporting premonitory symptoms [16-20]. This is a wide frequency range which can possibly be explained by the varying ways of data acquisition, the variable number of premonitory symptoms investigated and the different patient populations in the five studies. In contrast to these studies we only regarded symptoms with an onset of 2 hours and more prior to the headache as truly premonitory. The found prevalence rate in our study is thus located in the lower range of previously reported rates. The most frequent premonitory symptoms were a tense neck, phonophobia and difficulty concentrating and are thus in line with previous studies $[17,18,20]$.

As data were assessed retrospectively, a recall-bias might have led to an underestimation of triggers and premonitory symptoms. As however migraine patients have the migraine pain as a recall anchor (e.g. memory aid) and often pay special attention to possible triggers and premonitors, this attribution bias could have resulted in an over-estimation of the described phenomena. Additionally patients of a tertiary headache center are most likely more seriously afflicted by their migraine disorder 
and the associated symptoms than the general population of migraine sufferers. It is thus possible that the described distribution of symptoms and triggers might not reflect the average of migraine sufferers.

Our data show a clear association of the presence of certain symptoms in the premonitory phase of migraine and certain trigger factors corresponding to these symptoms, e.g. photophobia as a premonitory symptom and flickering or bright light as a corresponding trigger factor. The pronounced perception of certain factors as triggers might thus be merely a result of the presence of corresponding symptoms in the premonitory phase of migraine. The presence of e.g. photophobia, osmophobia or food craving might result in a pronounced perception of intense visual stimuli such as bright or flickering light as especially adverse and even pain inducing or in an increased intake of certain foods and thus lead to the conclusion in patients and physicians, that these factors can actually trigger migraine pain. As there is currently no study showing a reliable precipitation of migraine pain by typical trigger factors $[12,13]$, it seems reasonable to assume that so-called triggers are really just early symptoms of the migraine attack.

It is however also possible that these classic triggers are in fact able to precipitate migraine pain but not independently and at all times: There is currently a lot of evidence that certain parameters of neuronal function (such as cortical excitability, response to evoked potentials, BOLD-response to standardized stimuli, activity levels of different areas of the brain and brainstem) in migraine patients change during different stages of the migraine cycle [21-25]. One study even could show that the level of perceived stress increased during the four days preceding a migraine attack [26]. Moreover, for a certain factor to be perceived as a migraine trigger by patients, a close temporal relationship between exposure time and the onset of the pain phase is important. Thus it becomes quite likely, that commonly reported trigger factors are not independent precipitators of migraine pain, but that their ability to actually provoke attacks depends on the stage of the migraine cycle during which they are applied.

Our data further support the assumption that in some patients the typical accompanying symptoms of migraine pain actually precede the pain phase $[1,17-20,27]$ and thus offer additional evidence for the common assumption that a migraine attack is not just an isolated event of pain but it is more likely a continuous up and down of certain sensory and bodily functions on top of which comes the headache. If migraine is understood as a threshold problem, any external stimulus may or may not be perceived as a trigger, depending on the threshold level. The threshold itself oscillates and as long as it is above an individual mark a so-called "trigger" has no consequences. Once the threshold level falls below that mark, the incoming sensation will be perceived as unpleasant and misinterpreted as a trigger. However, the change in threshold is the original mechanism behind the origination of an attack and the growing unpleasantness of a usually inert external stimulus is probably nothing more than the first perception of the decreasing threshold. It probably depends on the susceptibility of a given migrainous individual whether the pronounced and possibly unpleasant perception of light or smell or other stimuli are the first symptom of the attack and photophobia, osmophobia, nausea or phonophobia will then be one of the distinctive following symptoms in the attack.

\section{Conclusions}

Our data corroborate the view that most of the reported common trigger factors of migraine are not so much independent triggers that at any time might precipitate an acute attack of migraine pain, but that they are most likely just misunderstood symptoms of the premonitory phase of migraine pain, which is already part of the migraine attack. Future studies investigating "migraine" and also "triggers" have to carefully assess the precise state of the migraine cycle, which must include not only the last but also the beginning of the next attack. We need to characterize the involved neuronal and autonomic subnetworks and their connections during all parts of the migraine cycle if we are ever to understand migraine.

\section{Competing interests}

The authors declare that they have no competing interests.

\section{Authors' contributions}

AM, TPJ and LHS collected the data. LHS analysed the data. LHS and AM drafted the manuscript. All authors read and approved the final version of the manuscript.

\section{Funding}

This work was supported by the German Research Foundation, SFB936/A5 (A.M.) and the 7th Framework EU-project EuroHeadPain (\#602633) to A.M.

Received: 4 December 2014 Accepted: 17 January 2015

Published online: 15 February 2015

\section{References}

1. Kelman $L$ (2007) The triggers or precipitants of the acute migraine attack. Cephalalgia 27(5):394-402

2. Peroutka SJ (2014) What turns on a migraine? A systematic review of migraine precipitating factors. Curr Pain Headache Rep 18(10):454

3. Spierings EL, Ranke AH, Honkoop PC (2001) Precipitating and aggravating factors of migraine versus tension-type headache. Headache 41(6):554-558

4. Al-Shimmery EK (2010) Precipitating and relieving factors of migraine headache in 200 iraqi kurdish patients. Oman Med J 25(3):212-217

5. Baldacci F, Vedovello M, Ulivi M, Vergallo A, Poletti M, Borelli P, Nuti A, Bonucelli U (2013) How aware are migraineurs of their triggers? Headache 53(5):834-837

6. Neut D, Fily A, Cuvellier J-C, Vallée L (2012) The prevalence of triggers in paediatric migraine: a questionnaire study in 102 children and adolescents. J Headache Pain 13(1):61-65 
7. Scharff L, Turk DC, Marcus DA (1995) Triggers of headache episodes and coping responses of headache diagnostic groups. Headache 35(7):397-403

8. Mollaoğlu M (2013) Trigger factors in migraine patients. J Health Psychol 18(7):984-994

9. Kelman $L$ (2004) Osmophobia and taste abnormality in migraineurs: a tertiary care study. Headache 44(10):1019-1023

10. Hay KM, Mortimer MJ, Barker DC, Debney LM, Good PA (1994) 1044 women with migraine: the effect of environmental stimuli. Headache 34(3):166-168

11. Yadav RK, Kalita J, Misra UK (2010) A study of triggers of migraine in India. Pain Med Malden Mass 11(1):44-47

12. Hougaard A, Amin F, Hauge AW, Ashina M, Olesen J (2013) Provocation of migraine with aura using natural trigger factors. Neurology 80(5):428-431

13. Marcus DA, Scharff L, Turk D, Gourley LM (1997) A double-blind provocative study of chocolate as a trigger of headache. Cephalalgia Int J Headache 17(8):855-862, discussion 800

14. Dahlem MA, Kurths J, Ferrari MD, Aihara K, Scheffer M, May A (2014) Understanding migraine using dynamic network biomarkers. Cephalalgia Int $J$ Headache. Epub ahead of print.

15. Goadsby PJ, Silberstein SD (2013) Migraine triggers Harnessing the messages of clinical practice. Neurology 80(5):424-425

16. Kelman $L$ (2004) The premonitory symptoms (prodrome): a tertiary care study of 893 migraineurs. Headache 44(9):865-872

17. Schoonman GG, Evers DJ, Terwindt GM, van Dijk JG, Ferrari MD (2006) The prevalence of premonitory symptoms in migraine: a questionnaire study in 461 patients. Cephalalgia Int J Headache 26(10):1209-1213

18. Quintela E, Castillo J, Muñoz P, Pascual J (2006) Premonitory and resolution symptoms in migraine: a prospective study in 100 unselected patients. Cephalalgia 26(9):1051-1060

19. Cuvellier J-C, Mars A, Vallée L (2009) The prevalence of premonitory symptoms in paediatric migraine: a questionnaire study in 103 children and adolescents. Cephalalgia Int J Headache 29(11):1197-1201

20. Giffin NJ, Ruggiero L, Lipton RB, Silberstein SD, Tvedskov JF, Olesen J, Altman J, Goadsby PJ, Macrae A (2003) Premonitory symptoms in migraine: an electronic diary study. Neurology 60(6):935-940

21. Stankewitz A, Aderjan D, Eippert F, May A (2011) Trigeminal nociceptive transmission in migraineurs predicts migraine attacks. J Neurosci Off J Soc Neurosci 31(6):1937-1943

22. Stankewitz A, May A (2007) Cortical excitability and migraine. Cephalalgia Int J Headache 27(12):1454-1456

23. Judit A, Sándor PS, Schoenen J (2000) Habituation of visual and intensity dependence of auditory evoked cortical potentials tends to normalize just before and during the migraine attack. Cephalalgia Int J Headache 20 (8):714-719

24. Weiller C, May A, Limmroth V, Jüptner M, Kaube H, Schayck RV, Coenen HH, Diener HC (1995) Brain stem activation in spontaneous human migraine attacks. Nat Med 1(7):658-660

25. Maniyar FH, Sprenger T, Monteith T, Schankin C, Goadsby PJ (2014) Brain activations in the premonitory phase of nitroglycerin-triggered migraine attacks. Brain J Neurol 137(Pt 1):232-241

26. Levor RM, Cohen MJ, Naliboff BD, McArthur D (1986) Psychosocial precursors and correlates of migraine headache. J Consult Clin Psycho 54(3):347-353

27. Maniyar FH, Sprenger T, Schankin C, Goadsby PJ (2014) Photic hypersensitivity in the premonitory phase of migraine-a positron emission tomography study. Eur J Neurol Off J Eur Fed Neurol Soc 21(9):1178-1183

\section{Submit your manuscript to a SpringerOpen ${ }^{\circ}$ journal and benefit from:}

- Convenient online submission

- Rigorous peer review

- Immediate publication on acceptance

- Open access: articles freely available online

- High visibility within the field

- Retaining the copyright to your article

Submit your next manuscript at $>$ springeropen.com 